\title{
Rules for predicting the conformational behavior of saturated seven-membered heterocycles
}

\author{
Antonio Entrena, Joaquín M. Campos, Miguel A. Gallo, and Antonio Espinosa*
}

Departamento de Química Farmacéutica y Orgánica, Facultad de Farmacia, Universidad de Granada, c/ Campus de Cartuja s/n, 18071 Granada, Spain

E-mail: aespinos@ugr.es

This paper is dedicated to Prof. Eusebio Juaristi on the occasion of his $55^{\text {th }}$ birthday

(received 10 Mar 05; accepted 15 Apr 05; published on the web 19 Apr 05)

\begin{abstract}
The systematization between the spatial orientation of the hydrogen atom being substituted in the base ring, the nature of the substituent, and the stability of the conformations of the ring allow the establishment of general principles related to the conformational behavior of saturated sevenmembered heterocycles.
\end{abstract}

Keywords: Anomeric effect, gauche effect, isoclinal orientation, methoxy-1,4-dioxepanes, saturated seven-membered heterocycles

\section{Contents}

1. Introduction

2. Nomenclature of Conformations in Seven-Membered Rings

3. Conformations of Saturated Seven-Membered Heterocycles

3.1 Oxepane

3.2 Thiepane

3.3 1,4-Oxathiepane and 1,4-Dioxepane

4. Conformational Behavior of Methoxy-1,4-Dioxepanes

4.1 5-Methoxy- and 2-Methoxy-1,4-Dioxepanes

4.2 6-Methoxy-1,4-Dioxepane

5. Orientation of Hydrogen Atoms

6. Conformational Behavior of 1,4-Dioxepane Derivatives

7. Conclusions

8. References 


\section{Introduction}

In recent years there has been a marked increase in the number of papers describing sevenmembered heterocycles but the conformational analysis is generally avoided because it is not an easy task. The expression of the anomeric and gauche effects does not follow the model for that of six-membered rings and so the systematization of general rules for the prediction of their conformational behavior is very advisable. This step forward could be specially useful to those researchers who know six-membered rings thoroughly and try to understand the peculiar geometry of seven-membered rings.

In contrast to the six-membered rings in which conformational studies are generally limited to chair conformations, the seven-membered cyclic systems are much more flexible. These rings occur in complex pseudorotational equilibria characterized by the existence of numerous conformations of similar energy and the absence of significant pseudorotational barriers. For all these reasons the study of such compounds is difficult using experimental techniques. Condensation with a benzene ring or the introduction of a double bond confers enough rigidity on the seven-membered moiety so as to freeze the pseudorotational equilibrium at a low temperature. In these cases, dynamic nuclear magnetic resonance has shown to be an appropriate tool in order to assess the most stable conformations and the energetic barriers shown in the equilibrium of such compounds. Several examples of benzene-fused or unsaturated sevenmembered heterocycles with one or two heteroatoms as benzoxepine, ${ }^{1-3} 4,7$-dihydro-2H-1,3dioxepine, ${ }^{4}$ or benzodioxepine ${ }^{5-8}$ derivatives have been studied by means of such a technique.

When the pseudorotational barriers are too low to be observed by means of NMR, theoretical methods are resorted to as powerful tools, especially molecular mechanics. ${ }^{9}$ Such a technique has been successfully used in the study of hydrocarbons (cycloheptane ${ }^{10}$ and cycloheptene $^{11}$ ), heterocycles with one heteroatom (oxepane, ${ }^{12}$ thiepane ${ }^{13}$ and perhydroazepine ${ }^{14}$ ) and heterocycles con two heteroatoms (1,3-dioxepane, ${ }^{15}$ 1,4-dioxepane ${ }^{16}$ and 1,4-oxathiepane $\left.{ }^{13}\right)$ and the cycloheptene analogs with two heteroatoms (1,4-dioxepines $\left.{ }^{17}\right)$.

The anomeric effect ${ }^{18 a, b}$ implies the preference for the synclinal (gauche) conformation of a fragment $\mathrm{C}-\mathrm{Y}-\mathrm{C}-\mathrm{X}-\mathrm{C}$, where $\mathrm{X}$ and $\mathrm{Y}$ are highly electronegative heteroatoms, such as $\mathrm{O}, \mathrm{N}$ or $\mathrm{F}$. It is responsible for the axial orientation of electronegative $\mathrm{C}-2$ substituents in six-membered heterocycles $^{19}$. On the other hand, the conformational preference of the X-C-C-Y moiety (also bearing electronegative groups such as $\mathrm{O}, \mathrm{F}$ or $\mathrm{CN}$ ) by a synclinal orientation is attributed to the gauche effect. ${ }^{18 \mathrm{~b}, 20}$ This effect is responsible for the greater stability of the gauche conformation of 1,2-dimethoxyethane $\mathrm{e}^{21}$ and polyoxyethylene $\mathrm{e}^{22}$ and for the axial orientation in 5-methoxy-1,3dioxane. $^{23}$

In order to facilitate the use of our methodology, we here present a short guide for predicting the conformational behavior of seven-membered rings with one or two heteroatoms that has been specially written for those who are interested in the practical application of our rules. Moreover, the nomenclature of conformations in seven-membered rings and the orientation of hydrogen atoms will be explained herein in order to facilitate the lecture and 
understanding of our guide. Nevertheless, those who are also interested in the fundamental problem may look for the original references cited at the end of this account.

For the sake of simplicity, this account is organized by substrate type. It begins with the nomenclature of conformations in seven-membered rings and the orientation of hydrogen atoms. A short set of conclusions is included at the end of the paper.

\section{Nomenclature of Conformations in Seven-Membered Rings}

Historically, cycloheptane ${ }^{10}$ was the first compound studied, and this has served as a model for subsequent investigations. Such a compound can adopt several significant conformations. First, a set of conformations that are named chairs $(\mathbf{C})$, which do not constitute real conformers due to the fact that the non-bonding interactions between hydrogen atoms destabilize them. The more stable conformers are the twist-chairs (TC), whilst the $\mathbf{C}$ forms constitute transition states in the pseudorotation process between two twist-chairs. Both conformations constitute what is called the chair/twist chair family (C/TC).

Secondly, another pseudorotating family exists (B/TB). Again, non-bonding interactions between hydrogen atoms turn the boat conformations into transition states of the pseudorotating process. TB conformations possess conformational energies higher than TC conformations in spite of being energetic minima. Finally, each TC conformation may be converted into a TB conformation by means of a pseudorotating process. So fourteen conformations of each energetically degenerated type exist. Figure 1 shows the four different conformations of cycloheptane.

Chair and boat conformations are characterized by the presence of a plane of symmetry whilst the TC and TB conformers present a binary axis. Such elements of symmetry bisect one bond of the ring and pass through the opposite carbon atom.

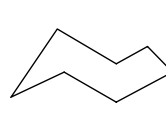

C

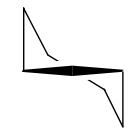

TC



B

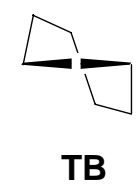

TB

Figure 1. Conformations of cycloheptane.

The energy differences between conformations are small, except for the interconversions between both pseudorotating families (TC $\leftrightarrow$ TB transition). The relative values of such energies depend on the force fields used for this study. The early force fields used by Hendrickson ${ }^{10 a}$ and Bocian $^{10 b}$ have a tendency to overestimate the energy differences between the $\mathbf{C}$ and TC conformations. Other force fields such as $\mathrm{MM}^{24}$ and MM3(92) ${ }^{25}$ give more concordant values with the scanty experimental data given by scientific literature. Experimental geometries and vibrational amplitudes ${ }^{26}$ agree well with those obtained from the MM2 ${ }^{10 \mathrm{c}}$ force field calculations. 
Several ways have been proposed to denote the different conformation of the ring, among them those of Hendrickson, ${ }^{27}$ Díez $^{28}$ and Foces-Foces. ${ }^{29}$ Nevertheless, we have proposed a new type of nomenclature to fully characterize each conformation based on the situation of the element of symmetry and on the sign of the intracyclic dihedral angles. ${ }^{30}$ This nomenclature is more complete and easier to use, and is represented in the Figure 2 for all the TC/C conformations of oxepane.

In the majority of conformations there does not exist an axis or a plane of symmetry due to the presence of the heteroatom. That is why we consider the existence of pseudoaxes or pseudoplanes with the aim to extend the nomenclature to any heterocycle. The following characteristics are noticed:

(1) $\mathrm{C} 1$ is the chair conformation in which the dihedral angle $\omega_{1234}$ (defined by the $\mathrm{O}_{1}-\mathrm{C}_{2}-\mathrm{C}_{3}-\mathrm{C}_{4}$ atoms) has a value of $0^{\circ}$ and the angle $\omega_{2345}$ is positive. The rest of the intracyclic dihedral angles alternate the value of their sign.

(2) Pseudorotation takes place in such a way that the pseudoelement of symmetry moves clockwise and hence, in $\mathbf{C} 2$ the angle $\omega_{2345}$ is $0^{\circ}$, whilst $\omega_{1234}$ has a positive value.

(3) The twist-chair TC1 is situated between $\mathbf{C 1}$ and C2 in the pseudorotational process, and the torsional angles $\omega_{1234}$ and $\omega_{2345}$ have positive signs. Twist-chairs are differentiated in the sign of an intracyclic angle. Thus, in order to go from TC1 to TC2 the sign of the dihedral angle $\omega_{2345}$ has to be changed, to go from TC2 to TC3 the sign of $\omega_{3456}$ has to be changed, etc. 


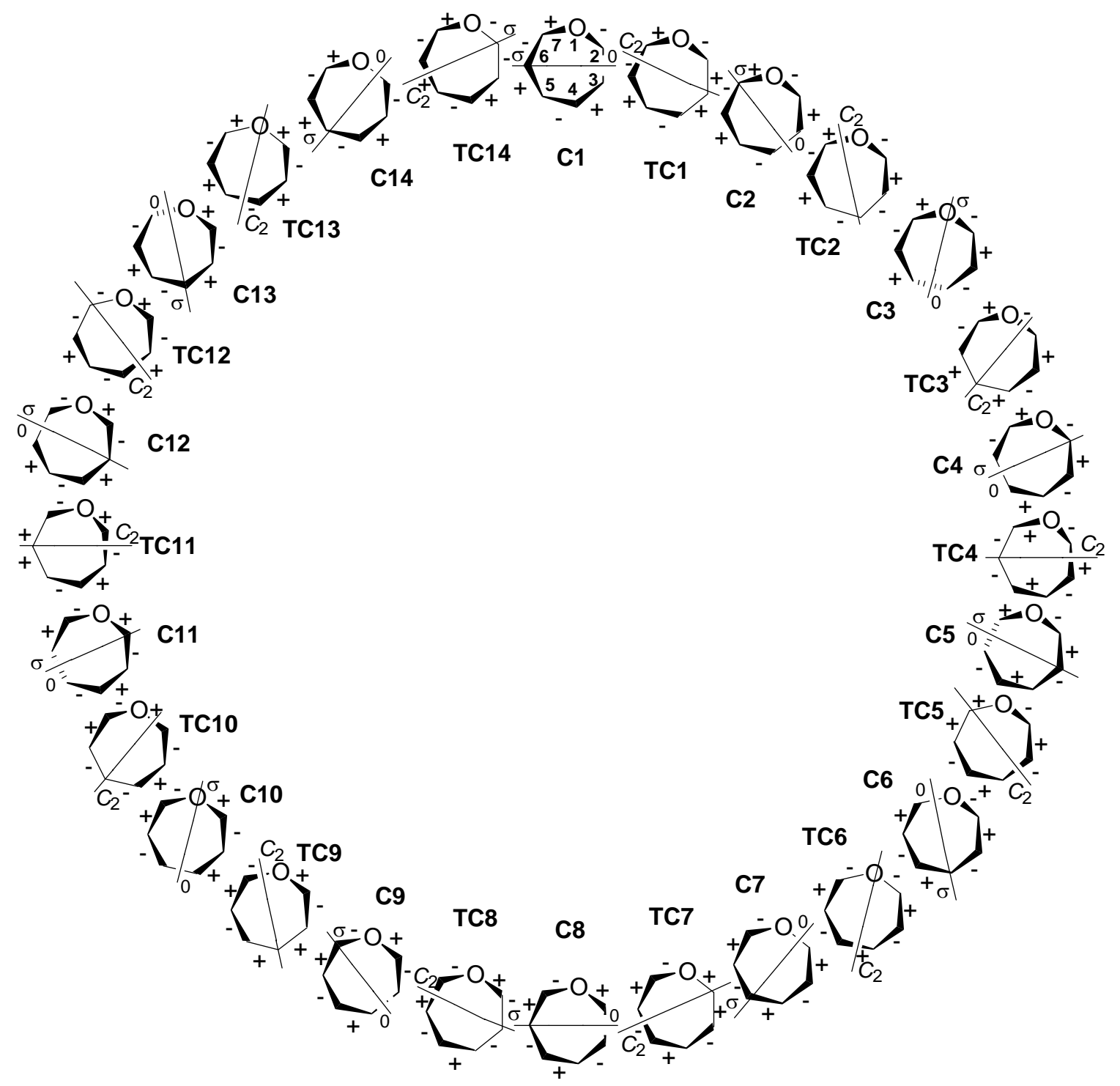

Figure 2. Representation of the pseudorotation in the C/TC family in oxepane. The notations $\sigma$ and $C_{2}$ denote the pseudoplane and pseudoaxis for each conformation, respectively.

As is logical, the presence of the heteroatom introduces some distortions in the geometries of the chair forms and the dihedral angles rarely acquire the value of zero degrees. Accordingly a chair is considered as such when any dihedral angle acquires a value sufficiently close to zero (in general, a value of $\pm 8^{\circ}$ spans the interval of all of our compounds. 


\section{Conformations of Saturated Seven-Membered Heterocycles}

\subsection{Oxepane}

The molecular dynamics of oxepane ${ }^{12}$ was extensively studied by means of the MM2 ${ }^{24}$ and MM3(92) ${ }^{25}$ force fields of Allinger, concordant results being obtained with both fields. Again, the twist-chair conformations are the true conformers for this heterocycle, and the energy differences are also explained as a function of non-bonding interactions between hydrogens. ${ }^{12}$ Table 1 summarizes the TC relative energies as well as the position of the $C 2$ pseudoaxis in each conformation. Even if both force fields agree well in the stability order of the four different conformers, the absolute value of energy depends on the program used for the calculation.

Table 1. Influence of the $\mathrm{O}$ and $\mathrm{S}$ atoms on the oxepane and thiepane TC conformation stability as a function of the position of $C 2$ pseudoaxis. Energies are calculated by means of both MM2 and MM3 force fields

\begin{tabular}{|c|c|c|c|c|c|c|}
\hline \multirow[t]{3}{*}{ Conformer } & \multirow[t]{3}{*}{$C 2$ position $^{\mathrm{a}}$} & \multirow[t]{3}{*}{ X-Effect ${ }^{b}$} & \multirow{2}{*}{\multicolumn{2}{|c|}{$\begin{array}{c}\text { Oxepane } \\
\text { Rel. E. }^{\mathrm{c}}\end{array}$}} & \multirow{2}{*}{\multicolumn{2}{|c|}{$\begin{array}{l}\text { Thiepane } \\
\text { Rel. E. }\end{array}$}} \\
\hline & & & & & & \\
\hline & & & $\mathrm{MM}^{12}$ & $\mathrm{MM}^{12}$ & $\mathrm{MM} 2^{13}$ & $\mathrm{MM}^{\mathrm{d}}$ \\
\hline TC1 (TC8) & 3 & $\beta$ & 1.01 & 0.75 & 0.00 & 0.00 \\
\hline TC2 (TC9) & 4 & $\gamma$ & 0.79 & 0.70 & 0.87 & 1.73 \\
\hline TC3 (TC10) & 5 & $\gamma$ & 0.79 & 0.70 & 0.87 & 1.73 \\
\hline TC4 (TC11) & 6 & $\beta$ & 1.01 & 0.75 & 0.00 & 0.00 \\
\hline TC5 (TC12) & 7 & $\alpha$ & 0.00 & 0.00 & 0.49 & 1.61 \\
\hline TC6 (TC13) & 1 & $\mathrm{X}$ & 1.34 & 1.58 & 0.93 & 2.26 \\
\hline TC7 (TC14) & 2 & $\alpha$ & 0.00 & 0.00 & 0.49 & 1.61 \\
\hline
\end{tabular}

${ }^{a}$ Atom number through which the $C 2$ pseudoaxis is passing in this conformation.

${ }^{\mathrm{b}}$ Situation of the heteroatom in relation to the $C 2$ pseudoaxis. The TC relative energy can be considered as a measure of the influence of the heteroatom over the stability of the TC, i. e. $\beta_{\mathrm{O}^{-}}$ effect means that the presence of an $\mathrm{O}$ atom in a $\beta$ position to the $C 2$ pseudoaxis increases the $\mathrm{TC}$ relative energy in $1.01 \mathrm{kcal} / \mathrm{mol}(\mathrm{MM} 2)$ or $0.75 \mathrm{kcal} / \mathrm{mol}(\mathrm{MM} 3)$.

${ }^{\mathrm{c}}$ Relative energy ( $\left.\mathrm{kcal} / \mathrm{mol}\right)$ is defined in relation to the most stable TC conformation.

${ }^{\mathrm{d}}$ A. Entrena, J. Campos, M. A. Gallo and A. Espinosa, unpublished results.

The most stable twist-chair conformers are those in which the hydrogen atoms of the carbon atoms $\mathrm{C}-2$ or $\mathrm{C}-7$ ( $\alpha$ to the heteroatom) are isoclinal (TC5, TC7, TC12 and TC14), i. e., C-2 or C-7 are on a two-fold axis.

Both force fields indicates that the most energetic conformers are those with the $C 2$ axis passing through the heteroatom (TC6, TC13); MM3 assigns a slightly higher value to the relative energy. 
Finally, TC conformations with the two-fold axes situated in a $\gamma$ position to O (TC2, TC3, TC9, TC10) are more stable than those with the $C 2$ axis on the $\beta$ one (TC2, TC9, TC6, TC13).

The relative energy of each one of these TC conformations can be used to describe how the position of the two-fold axis in relation to the $\mathrm{O}$ atom influences the stability of the TC conformation. For example, when the $C 2$ axis passes through the $C-2$ atom ( $\alpha$-position) the resulting TC is the more favored one; when passing through the $\mathrm{O}-1$ atom, the TC is destabilized by 1.38 or $1.58 \mathrm{kcal} / \mathrm{mol}$ depending on the force field considered. We refer to these values as $\mathrm{O}_{\alpha}-, \mathrm{O}_{\beta}-, \mathrm{O}_{\gamma}-$ and $\mathrm{O}_{0}$-effects and we will see later that they have an almost additive meaning, and are useful to qualitatively predict the TC stability of seven-membered heterocycles. Figure 4 shows a schematic representation of these effects as a function of the pseudoaxis position.

\subsection{Thiepane}

The molecular dynamics of thiepane ${ }^{13}$ was amply studied by means of MM2 molecular mechanics, ${ }^{24}$ showing a conformational preference for the twist chair forms in the ground state. The interactions arising from the hydrogen atoms are the ones that most influence the energy of the conformations. ${ }^{13}$

The most stable conformations are those that present the $C 2$ pseudo-axis passing through the C-2 (TC2, TC9) or C-5 (TC6, TC13) carbon atoms, situated in a $\beta$ position in relation to the heteroatom. On the contrary, the most energetic conformers are TC6 and TC13, with the sulfur atom situated on the binary pseudo-axes. Finally, conformations with the $C 2$ axis on position $\alpha$ (TC5, TC7, TC12, TC14) seems to be slightly more stable than those on $\gamma$ position (TC2, TC3, TC9, TC10).

When MM3 force field is used to calculate relative energies, the stability order of the four different TC conformations remains unchanged. Nevertheless, the absolute values of the relative energies are higher than those calculated by means of MM2.

In this compound, similarly to oxepane, relative energies can be used to describe how the position of the $\mathrm{S}$ atom in relation to the two fold axes affects the stability of the TC conformation. In this case, we can refer to these values as $\mathrm{S}_{\alpha}-, \mathrm{S}_{\beta}-, \mathrm{S}_{\gamma}-$ and $\mathrm{S}_{0}$-effects that also show a predictive meaning. Figure 4 also represents schematically the S-effects for thiepane. 


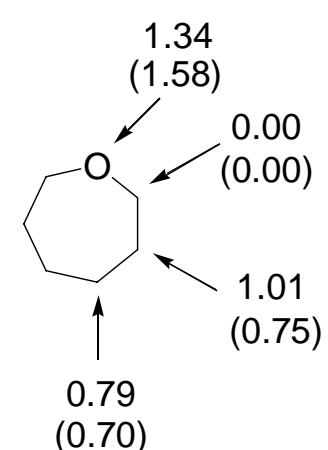

Oxepane

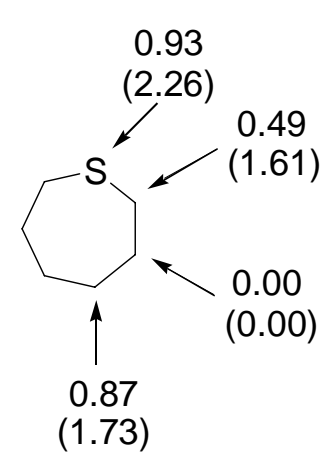

Thiepane

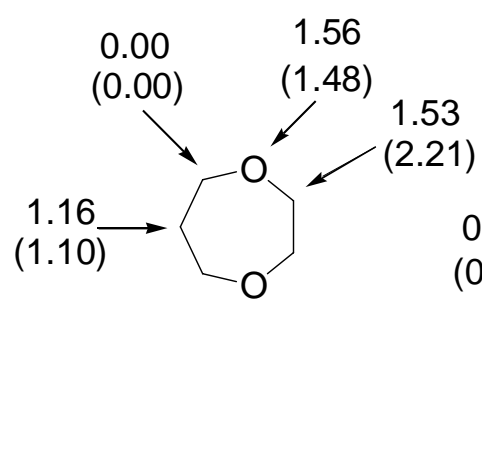

1,4-Dioxepane

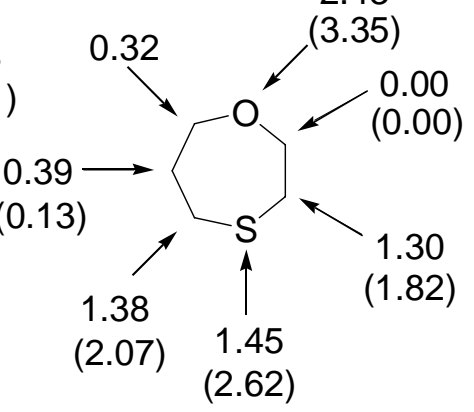

1,4-Oxathiepane

Figure 4. Schematic representation of the relative energy $(\mathrm{kcal} / \mathrm{mol})$ of TC conformations as a function of the position of the $C_{2}$ axis, calculated by means of the MM2 force field. Energies calculated by means of the MM3 force field are indicated in parentheses.

\subsection{1,4-Oxathiepane and 1,4-dioxepane}

As has been mentioned above, it is possible to qualitatively determine the most stable conformations for 1,4-dioxepane and 1,4-oxathiepane from the relative energies of oxepane and thiepane. In these cases an almost additive effect exists between both heteroatoms.

Table 2 shows the relative energies for the TC conformations of 1,4-oxathiepane as well as the position of the pseudoaxes. In each conformation, every one of the heteroatoms affects the stability of the TC in a value that depends on its position in relation to the $C_{2}$ axis. These influences can be considered similar to those previously described for oxepane and thiepane. Table 2 refers to these effects as the $\mathrm{O}_{1}$ - and $\mathrm{S}_{4}$-effect and the sum of both effects can be used to estimate which would be the stability order of TC conformations. For example, in the TC1 conformation the two-fold axis passes through C-3 atom and hence O-1 exerts an $\mathrm{O}_{\beta}$ - effect while S-4 shows an $\mathrm{S}_{\alpha}$-effect. Since the value of these effects depends on the force field used (Table 1), the expected stability order (EO) also depends on the force field employed. Hence, the O- and S-effects calculated using the MM2 (or MM3) force fields in oxepane and thiepane must be compared with the relative energies calculated using the same force field.

Table 2 shows that both force fields are successful in estimating the order of stability of the 5TC (or the 7TC) conformations of 1,4-oxathiepane. Only the TC1 and TC3 orders are inverted and the energy difference between both conformations is very small.

TC5 and TC4 are the second and third conformations. In the first one, O- 1 is $\alpha$ while S-4 is $\gamma$, whilst both heteroatoms in TC4 are in position $\beta$ in relation to the C2-axis. That is, both conformations are stabilized by the most favored situation of the oxygen (TC5) and the sulfur (TC4) atom. 
Table 2. Expected and found stability order for the TC conformations for 1,4-oxathiepane calculated by means of MM2 and MM3 force fields

\begin{tabular}{|c|c|c|c|c|c|c|c|c|c|c|}
\hline \multirow[t]{2}{*}{ Conformer } & \multirow{2}{*}{$\begin{array}{c}C 2 \\
\text { position }\end{array}$} & \multirow{2}{*}{$\begin{array}{l}\mathrm{O}_{1} \text {-effect, } \\
\mathrm{S}_{4} \text {-effect }\end{array}$} & \multicolumn{4}{|c|}{$\mathrm{MM} 2^{13}$} & \multicolumn{4}{|c|}{$\mathrm{MM}^{\mathrm{a}}$} \\
\hline & & & $\mathrm{O}_{1}+\mathrm{S}_{4}$ & $\mathrm{EO}^{\mathrm{b}}$ & Rel. E. & $\mathrm{SO}^{\mathrm{d}}$ & $\mathrm{O}_{1}+\mathrm{S}_{4}$ & $\mathrm{EO}^{\mathrm{b}}$ & Rel. E. & $\mathrm{SO}^{\mathrm{d}}$ \\
\hline TC1 (TC8) & 3 & $\beta, \alpha$ & 1.50 & 5 & 1.30 & 4 & 2.35 & 5 & 1.82 & 4 \\
\hline TC2 (TC9) & 4 & $\gamma, \mathrm{S}$ & 1.72 & 6 & 1.45 & 6 & 2.95 & 6 & 2.62 & 6 \\
\hline $\begin{array}{c}\text { TC3 } \\
(\mathrm{TC} 10)\end{array}$ & 5 & $\gamma, \alpha$ & 1.28 & 4 & 1.38 & 5 & 2.30 & 4 & 2.07 & 5 \\
\hline $\begin{array}{c}\text { TC4 } \\
(\mathrm{TC} 11)\end{array}$ & 6 & $\beta, \beta$ & 1.01 & 3 & 0.39 & 3 & 0.74 & 2 & 0.13 & 2 \\
\hline $\begin{array}{c}\text { TC5 } \\
(\mathrm{TC} 12)\end{array}$ & 7 & $\alpha, \gamma$ & 0.87 & 2 & 0.32 & 2 & 1.73 & 3 & 1.00 & 3 \\
\hline $\begin{array}{c}\text { TC6 } \\
(\mathrm{TC} 13)\end{array}$ & 1 & $\mathrm{O}, \gamma$ & 2.21 & 7 & 2.43 & 7 & 3.99 & 7 & 3.35 & 7 \\
\hline $\begin{array}{c}\text { TC7 } \\
(\mathrm{TC} 14)\end{array}$ & 2 & $\alpha, \beta$ & 0.00 & 1 & 0.00 & 1 & 0.00 & 1 & 0.00 & 1 \\
\hline
\end{tabular}

${ }^{a}$ A. Entrena, J. Campos, M. A. Gallo and A. Espinosa, unpublished results

${ }^{b}$ EO: Expected stability order as calculated by the addition of $\mathrm{O}_{1}$ and $\mathrm{O}_{4}$ effects

${ }^{c}$ Relative energies are defined in relation to the most stable conformation of the molecule.

${ }^{\mathrm{d}}$ SO: Stability order as found by MM2 and MM3 force field calculations and defined by the relative energy of the TC conformation.

The most stable conformer of this molecule is TC7, where the $\mathrm{O}$ and $\mathrm{S}$ atoms are situated $\alpha$ and $\beta$ in relation to the $C 2$-axes, respectively, the most favored situations in oxepane and thiepane.

TC2 and TC6 are the two most energetic conformers and in both conformations, the twofold axes pass through one heteroatom ( $\mathrm{S}$, and $\mathrm{O}$, respectively) and, consequently, the other heteroatom is in a $\gamma$ position. Since these are the situations less favored for both heteroatoms, in both conformations, a cooperative destabilization there exists and they are the most energetic ones of this molecule.

Finally, in TC1 and TC3 a $\mathrm{S}_{\alpha}$-effect is present in both conformations while the oxygen atom shows an $\mathrm{O}_{\beta}$ - and $\mathrm{O}_{\gamma}$-effect, respectively. The calculated relative energies indicate an order in stability that it is the opposite to the one expected from the addition of the influences of both heteroatoms. Nevertheless, the difference in the $\beta$ and $\gamma$-effects calculated in oxepane is very small in both force field and this discrepancy can be accepted.

Table 3 shows the relative energies of the TC conformations of 1,4-dioxepane. Only four different conformations have been found due to the symmetry of the ring. The most stable conformer is TC3 (TC5, TC10, TC12) in which the two-fold axis is situated on C-5 (or C-7) 
atom and is $\alpha$ in relation to one $\mathrm{O}$ atom and $\gamma$ to the other one. The $\alpha$-position is the most favored one in oxepane while the energy due to the $\mathrm{O}_{\mathrm{g}}$-effect is small.

Table 3. Expected and found stability order for the TC conformations of 1,4-dioxepane calculated by means of MM2 and MM3 force fields

\begin{tabular}{|c|c|c|c|c|c|c|c|c|c|c|}
\hline \multirow[t]{2}{*}{ Conformer } & \multirow{2}{*}{$\begin{array}{c}C 2 \\
\text { position }\end{array}$} & \multirow{2}{*}{$\begin{array}{l}\mathrm{O}_{1} \text {-effect, } \\
\mathrm{O}_{4} \text {-effect }\end{array}$} & \multicolumn{4}{|c|}{$\mathrm{MM} 2^{16}$} & \multicolumn{4}{|c|}{$\mathrm{MM3}^{30}$} \\
\hline & & & $\mathrm{O}_{1}+\mathrm{O}_{4}$ & $\mathrm{EO}^{\mathrm{a}}$ & Rel. E. & $\mathrm{SO}^{\mathrm{c}}$ & $\begin{array}{c}\mathrm{O}_{1}+ \\
\mathrm{O}_{4}\end{array}$ & $\mathrm{EO}^{\mathrm{a}}$ & Rel. E. & $\mathrm{SO}^{\mathrm{c}}$ \\
\hline TC1 (TC8) & 3 & $\beta, \alpha$ & 1.01 & 2 & 1.53 & 3 & 0.74 & 2 & 2.21 & 4 \\
\hline TC2 (TC9) & 4 & $\gamma, \mathrm{O}$ & 2.13 & 4 & 1.56 & 4 & 2.27 & 4 & 1.48 & 3 \\
\hline TC3 (TC10) & 5 & $\gamma, \alpha$ & 0.79 & 1 & 0.00 & 1 & 0.69 & 1 & 0.00 & 1 \\
\hline TC4 (TC11) & 6 & $\beta, \beta$ & 2.02 & 3 & 1.16 & 2 & 1.48 & 3 & 1.10 & 2 \\
\hline TC5 (TC12) & 7 & $\alpha, \gamma$ & 0.79 & 1 & 0.00 & 1 & 0.69 & 1 & 0.00 & 1 \\
\hline TC6 (TC13) & 1 & $\mathrm{O}, \gamma$ & 2.13 & 4 & 1.56 & 4 & 2.27 & 4 & 1.48 & 3 \\
\hline TC7 (TC14) & 2 & $\alpha, \beta$ & 1.01 & 2 & 1.53 & 3 & 0.74 & 2 & 2.21 & 4 \\
\hline
\end{tabular}

${ }^{a}$ Relative energies are defined in relation to the most stable conformation of the molecule.

${ }^{b}$ EO: Expected stability order as calculated by the sum of $\mathrm{O}_{1}$ and $\mathrm{O}_{4}$ effect

${ }^{c}$ SO: Stability order as found by MM2 and MM3 force field calculations and defined by the relative energy of the TC conformation.

When using the MM2 force field, the most energetic conformations are also identified. In TC2 (TC6, TC9 and TC13) the C2-axis contains one of the two oxygen atoms, while the other one is situated in a $\gamma$ position. The second and third conformations are also not correctly predicted. MM3 failed in the prediction for the other three conformations.

This behavior may be explained by the fact that substitution in the oxepane of one carbon atom by an additional oxygen atom not only diminishes the number of hydrogen atoms but also introduces two $\mathrm{CO}$ bonds in the ring. Since the $\mathrm{C}-\mathrm{O}$ bond length is smaller than the $\mathrm{C}-\mathrm{C}$ bond length, non-bonded interactions between hydrogen atoms are reinforced due to the smaller distance between them. Hence, an effect due to the presence of the two heteroatoms must be considered. On the other hand, since the C-S bond is considerably longer than the $\mathrm{C}-\mathrm{C}$ bond, in 1,4-oxathiepane such an effect due to the presence of two heteroatoms is considerably smaller and the predictions of the TC stability order are more suitable. 


\section{Conformational Behavior of Methoxy-1,4-Dioxepanes}

\subsection{5-Methoxy- and 2-methoxy-1,4-dioxepanes}

Once the base ring is studied, the substitution of the appropriate hydrogen atoms in the several conformations of the cycle will allow the study of the corresponding derivatives. Normally, only the twist-chair conformations need to be considered since, in all the cases we have studied, we have found that the presence of TB conformations in the conformational equilibria is not significant. The reason may be the great folding of boat conformations in 1,4-dioxepane and the high non-bonded interactions that arise when a bulky substituent is introduced. Moreover, 14 TC conformations there exist with an appreciably inferior energy to those of the $\mathbf{T B}$, and at least in one of these conformations the substituents will probably lodge in a way that their interactions with the ring are minimized.

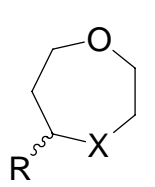

1: $X=O, R=O M e$
4: $X=\mathrm{CH}_{2}, \mathrm{R}=\mathrm{Me}$
7: $\mathrm{X}=\mathrm{CH}_{2}, \mathrm{R}=\mathrm{OMe}$

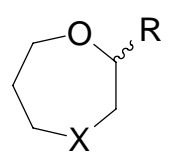

2: $\mathrm{X}=\mathrm{O}, \mathrm{R}=\mathrm{OMe}$

5: $\mathrm{X}=\mathrm{CH}_{2}, \mathrm{R}=\mathrm{Me}$

8: $\mathrm{X}=\mathrm{CH}_{2}, \mathrm{R}=\mathrm{OMe}$



3: $\mathrm{X}=\mathrm{O}, \mathrm{R}=\mathrm{OMe}$

6: $\mathrm{X}=\mathrm{CH}_{2}, \mathrm{R}=\mathrm{Me}$

9: $\mathrm{X}=\mathrm{CH}_{2}, \mathrm{R}=\mathrm{OMe}$

The first derivatives studied were the methoxy-1,4-dioxepanes 1-3. ${ }^{31}$ In the first two molecules the anomeric effect might play an important role in determining their conformational behavior. In the third one, gauche effect must control the TC conformation stability.

For compound 1 four significant conformations were found, especially TC3 whose conformational population was nearly $80 \%$. Only one (TC9) out of the four belongs to a group of conformations different from the most stable ones of the ring. It is important to emphasize that in TC3 the methoxy group does not adopt an axial orientation, contrary to what commonly occurs in six-membered rings. A partial stabilization of the twist-boat with an axial methoxy group was observed only in TC9. On the other hand, the methoxy group adopts an equatorial orientation in the other two conformations (Figure 5). Both MM2 and MM3 force fields agree well in the identification of the most stable conformer and in the order of stability of the different TC conformations. 


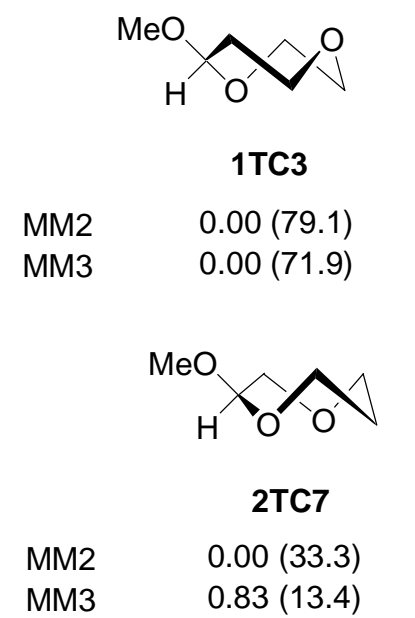

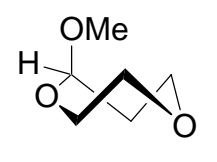

1TC9
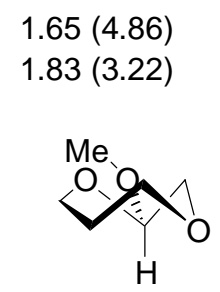

2TC5

0.11 (27.7) 0.00 (54.6)

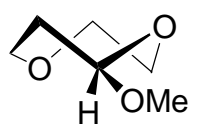

$1 \mathrm{TC} 12$

1.67 (4.66)

1.00 (13.28)

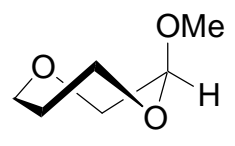

2TC10

0.22 (23.1)

1.04 (9.33)

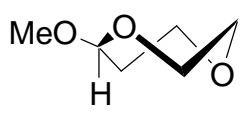

1TC10

1.81 (3.69)

1.58 (4.91)

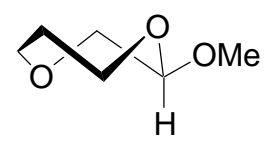

2TC3

$0.86(6.6)$

$0.78(14.6)$

Figure 5. The four most stable conformers calculated for compounds 1 and 2. MM2 and MM3 relative energies (kcal/mol) and conformational populations (in parentheses, \%) are shown.

In compound 2, MM2 and MM3 results differ in the identification of the most stable conformer. Nevertheless, two characteristics of the conformational behavior of this compound must be mentioned. The first one is that the 2-OMe group does not adopt an axial orientation as would be expected from the stabilization due to the anomeric effect. (Only in the third conformation an axial methoxy group was found). The second is the stabilization of the TC7 conformation that belongs to a group of a high energy TC conformation. MM2 identified TC7 as the most stable conformer of compound 3, although the relative energy of TC7 in 1,4-dioxepane is $1.53 \mathrm{kcal} / \mathrm{mol}$. MM3 also shows a significant stabilization of TC7, since its relative energy in 1,4-dioxepane is $2.21 \mathrm{kcal} / \mathrm{mol}$. It can be concluded that the anomeric effect can produce a change in the conformational preference of the base ring, and this is a consequence of the anomeric effect that is not observed in six-membered rings.

\subsection{6-Methoxy-1,4-dioxepane}

Finally, the four most stable conformations of compound $\mathbf{3}$ correspond with the more stable ones of the base ring. The gauche effect stabilizes an axial orientation of the methoxy group.

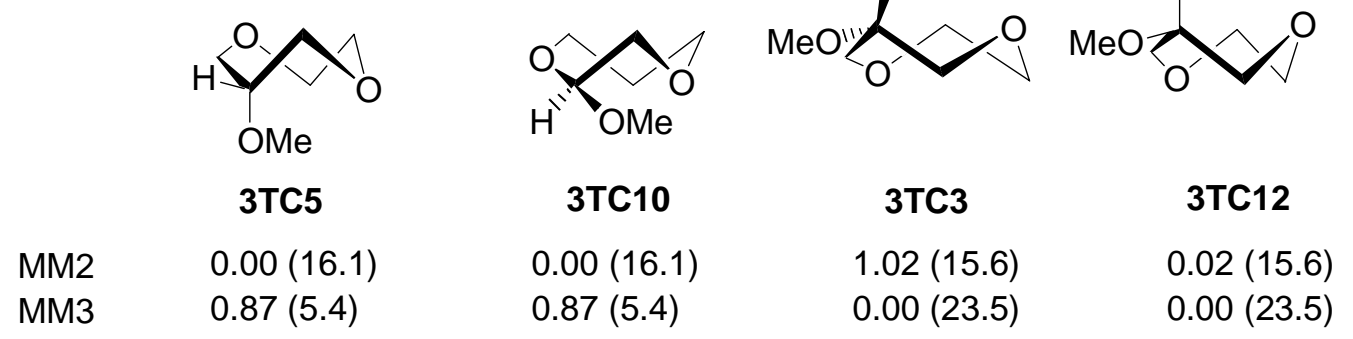

Figure 6. The four most stable TC conformers calculated for compound 3. MM2 and MM3 relative energies ( $\mathrm{kcal} / \mathrm{mol}$ ) and conformational populations (in parentheses, \%) are shown. 
Similarly to the previous compounds (1 and 2), a different degree of stabilization of the axial methoxy group can be observed depending on the force field used (Figure 6).

At this point, the following two important questions are posed:

(a) The first one is the reason why the anomeric effect does not force a substituent such as the methoxy group to be placed in a clearly axial position.

(b) The second one is the reason why the change in the conformational preference of the base ring takes place.

Both questions can be summarized in the following one: Given a saturated heptagonal heterocycle in which a substituent is introduced, would it be possible to establish general rules that allow the prediction of the conformational behavior of the ring? In order to answer this question we carried out a more systematic study on model compounds such as the oxepane derivatives 4-9. In such a study we tackle two important issues:

1) The first one was to determine and to correctly define the type of orientation that adopts a substituent in the ring.

2) Secondly, the relationship that exists between the orientation and the stability of the ring.

\section{Orientation of Hydrogen Atoms}

In addition to the axial and equatorial orientations, the hydrogen atoms in cycloheptane can be located in an isoclinal position. ${ }^{32}$ The relationship between the hydrogen orientations and the sign and magnitude of the endocyclic torsional angles that flank them is well known. ${ }^{33}$ Viewed clockwise, a $\beta$-orientated hydrogen atom is axial if the $\alpha_{1}$ and $\alpha_{2}$ torsion angles have the signs $(+,-),(0,+)$, or $(0,-)$, and is equatorial if the signs are $(-,+),(+, 0)$, or $(-, 0)$ (Figure 7$)$. Finally, such a hydrogen atom is isoclinal if the two torsion angles are equal in sign and magnitude.

Nevertheless, this criterion is not enough for the classification of the hydrogen atoms of heterocyclic systems. In oxepane, hydrogen atoms that are clearly axial (angles,+-$)$ or equatorial $(-,+)^{30}$ are found. Moreover, it is observed that hydrogen atoms exist in which the two torsional angles have equal signs, though with a different magnitude and, hence, they cannot be strictly considered as isoclinal according to Hendrickson's criteria. The magnitude difference depends on the position of hydrogen atoms in the ring. So, in $\mathrm{H}-4$ the difference is small, but is greater for H-2 and H-3. These differences may obviously be attributed to the distortion caused by the heteroatom, but this difference of the values is not the only important aspect. As we will see next, the two isoclinal hydrogen atoms are no longer equivalent and their substitution leads to distinct effects in the behavior of the ring.

Therefore the signs of the intracyclic torsional angles are not an adequate criterion to classify the hydrogen atoms. The classification is more precise if the torsional angles formed by the hydrogen atom, the atom to which it is linked and the two atoms that precede and follow it in the ring ( $\beta$ angles, Figure 7 ) are also considered. ${ }^{30}$ According to this criteria, the following types of hydrogen atoms can be considered in a seven-membered heterocycle. 


\section{Axial hydrogen atoms}

A hydrogen atom is considered axial when both $\beta$ angles have a value close to $60^{\circ}$. The substitution of such atoms modifies the statibility of the TC conformation depending on both the nature of the sustituent and the position of the hydrogen atom.

With the aim to estimate the value of the substituent influence on the stability of the TC conformations, we define the $\Delta \mathrm{E}$ value as the difference between the relative energy of the same TC conformation in the derivative and in oxepane. This value is not quantitative in the sense that it deals with a difference between relative energies of two different compounds (with distinct ranges of relative energy) and so its physical meaning is very limited, but will be very useful in the following discussion. $\Delta \mathrm{E}$ values for a Me group (Table 4) were developed from the study of methyloxepane derivatives 4-6, while the same values for the $\mathrm{MeO}$ were calculated from the methoxy analogs 7-9. ${ }^{30}$

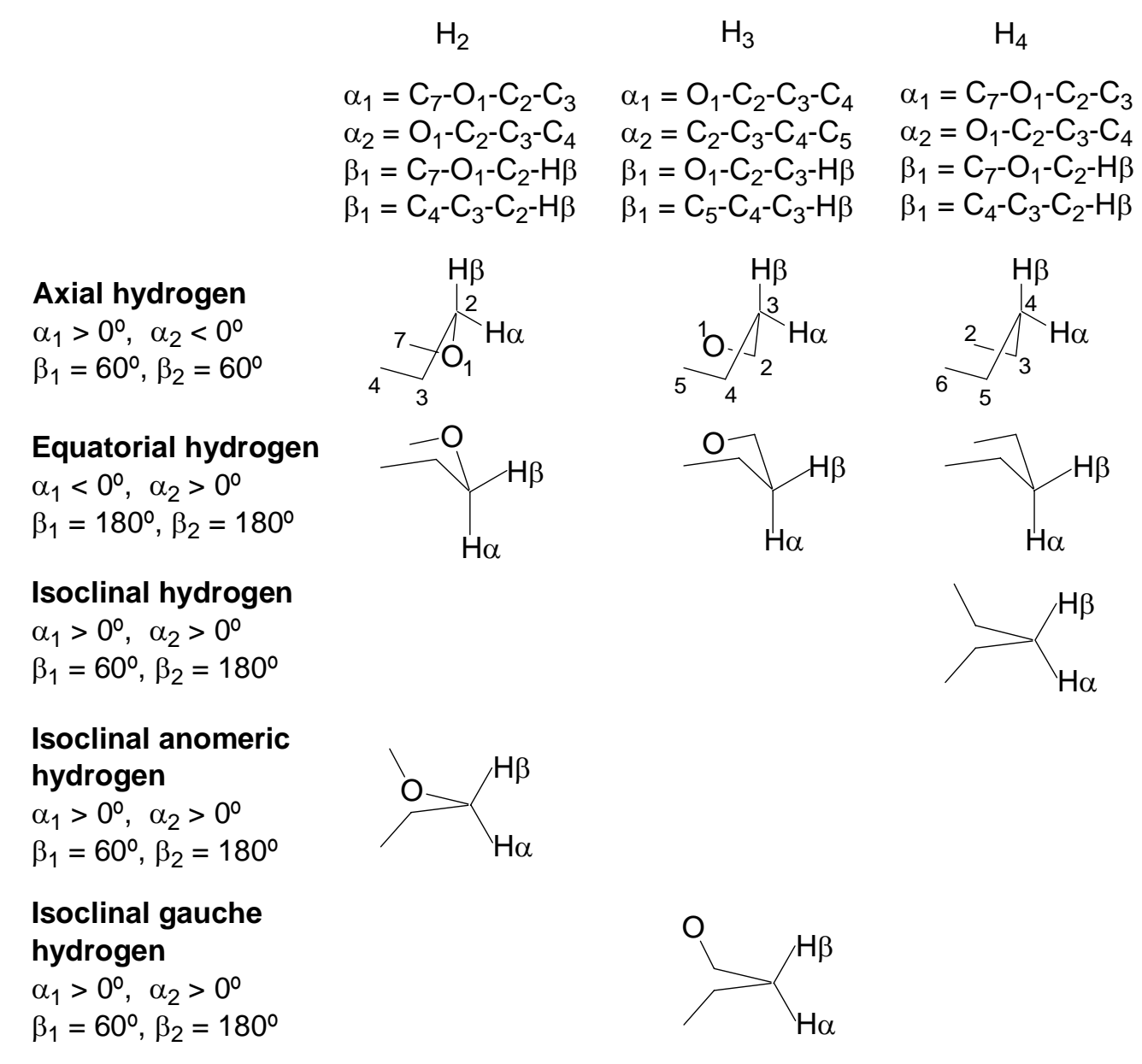

Figure 7. Hydrogen character of H-2, H-3 and H-4 hydrogen atoms of oxepane, defined as a function of both $\alpha$ and $\beta$ angle types. 
When C-4 is substituted, the ring oxygen atom is distant from the substituent and only steric effects appear, independently of the nature of the substituent. It can be observed that the methyl group introduces a higher destabilization than the methoxy group (Table 4). This result agrees with the free-energy difference between equatorial and axial methyl and methoxy groups in cyclohexane are 1.74 and $0.75 \mathrm{kcal} / \mathrm{mol}$, respectively. ${ }^{34}$

The substitution of an axial hydrogen in C-3 by a methyl group also leads to a destabilization of the TC conformation, although to a lesser extent than a 4-methyl group. This is due to the fact that steric interations take places with O-1 in the oxygen atom that only has two electronic lone pairs. On the other hand a 3-methoxy group does not introduce an important destabilization, since the gauche effect that appears between the two oxygen atoms would stabilize this orientation.

Table 4. Conformational steric energy $(\Delta \mathrm{E})$ for $\mathrm{Me}$ and $\mathrm{MeO}$ groups in 1,4-dioxepane calculated by means of the MM3 force field.

\begin{tabular}{ccccccc}
\hline \multicolumn{5}{c}{$\mathrm{Me}$} \\
\hline Orientation & $\mathrm{C}-2$ & $\mathrm{C}-3$ & $\mathrm{C}-4$ & $\mathrm{C}-2$ & $\mathrm{C}-3$ & $\mathrm{C}-4$ \\
$\mathbf{a}$ & $3.49-4.62$ & $1.17-2.60$ & $1.64-2.69$ & $1.1-4.3$ & $0.0-1.8$ & $0.3-1.8$ \\
$\mathbf{e}$ & $0.0-0.5$ & $0.0-0.4$ & $0.0-0.4$ & $0.4-1.7$ & $-0.2-0.31$ & $-0.3-0.3$ \\
$\mathbf{i}$ & & & $0.6-0.7$ & & & $0.0-0.4$ \\
$\mathbf{i}_{\mathbf{a}}$ & 1.4 & & & 0.0 & & \\
$\mathbf{i}_{\mathbf{g}}$ & & 0.3 & & & -0.5 & \\
$\mathbf{i}_{\mathbf{f}}$ & 0.7 & 0.6 & & 1.9 & 0.5 & \\
\hline
\end{tabular}

Finally, when the substitution takes place in C-2, the steric interactions of the axial methyl group are sensibly greater due to the shorter length of the $\mathrm{C}-\mathrm{O}$ bond and to the lesser $\mathrm{C}-\mathrm{O}-\mathrm{C}$ bond angle. On the other hand, a 2-methoxy group would be stabilized by the anomeric effect and the $\Delta \mathrm{E}$ value is sensibly smaller than the corresponding for a 2-methyl group. Nevertheless the steric interactions are still very important and, for this reason, an axial orientation is not the most favored for a 2-methoxy group.

\section{Equatorial hydrogen atom}

A hydrogen atom is equatorial when both $\beta$ angles acquire values close to $180^{\circ}$. The steric interactions that appear are small and the conformations will be fairly stable. In fact, $\Delta \mathrm{E}$ values for a methyl group vary between 0.0 and $0.5 \mathrm{kcal} / \mathrm{mol}$ in the three substitution positions, indicating a similar contribution of steric effects when substitution takes place. Nevertheless, the gauche and the anomeric effects destabilize these orientations when substitution by a MeO group takes place in C-3 and C-2, respectively, and a competence between steric and stereolectronic effect can be observed. In C-3, a slight stabilization occurs due mainly to a smaller steric interactions even if the gauche effect does not favor this orientation. In C-2 a clear destabilization of the equatorial orientation can be observed, mainly due to the anomeric effect 
even if the steric interactions are very low. From the $\Delta \mathrm{E}$ values we can conclude that the derivative conformations are of a similar stability to those of oxepane, in all the cases except in the 2-methoxy derivative, in which the equatorial orientation is not favored

\section{Isoclinal hydrogen atom}

The "pure" isoclinal hydrogen atoms appear only in the C-4 atom and in this case the $\alpha$ angles have equal signs and very similar absolute values. Moreover, one of the $\beta$ angles has a value close to $60^{\circ}$ and the other one to $180^{\circ}$, both being of the C-C-C-H type. The hydrogen with a $\beta$ orientation and its geminal one are perfectly equivalent and the steric interactions are intermediate between those of axial and of equatorial ones.

Nevertheless, in C-2 and C-3 a "pure" isoclinal orientation does not exist since in these cases the equivalence between both geminal hydrogen atoms disappears. The gauche isoclinal $\left(\mathrm{i}_{\mathrm{g}}\right.$ ) and false isoclinal $\left(\mathrm{i}_{\mathrm{f}}\right.$ ) orientations appear when the C-3 carbon atom is situated in the binary axis. In the former $\left(\mathrm{i}_{\mathrm{g}}\right)$ the torsional angle that adopts a value close to $60^{\circ}$ is of an O-C-C-H type. In this orientation a methyl group generates inferior steric interactions to those of an isoclinal methyl group giving a $\Delta \mathrm{E}$ value of $0.32 \mathrm{kcal} / \mathrm{mol}$, similarly to the situation observed for an axial hydrogen in $\mathrm{C} 3$ and $\mathrm{C} 4$. Furthermore, the methoxy group gives rise to specially stable conformations because the two following conditions are fulfilled: the steric interactions are small and the O-C-C-O torsional angle adopts a gauche disposition, and, hence, is stabilized by the gauche effect.

In the geminal hydrogen, the angle whose value is $60^{\circ}$ is of $\mathrm{C}-\mathrm{C}-\mathrm{C}-\mathrm{H}$ type and, when this happens, steric interactions are similar to those of an isoclinal 4-methyl group. Nevertheless, when the 3-MeO group adopts this orientation, there is no stabilization due to the gauche effect. Due to the different nature of the $\beta$ torsional angles involved in the interactions, the substitution of one or the other of the hydrogen atoms has different consequences in the greater or lesser stability of the ring. For this reason, this hydrogen has been named false isoclinal $\left(\mathrm{i}_{\mathrm{f}}\right)$ and the $\Delta \mathrm{E}$ values are 0.59 and $0.51 \mathrm{kcal} / \mathrm{mol}$ for $\mathrm{Me}$ and $\mathrm{MeO}$ groups. An experimental confirmation for the great preference of the $\mathbf{i}_{\mathbf{g}}$ orientation for the methoxy groups in seven-membered rings is offered by St. Jacques et al. ${ }^{1}$ in 1-benzoxepine derivatives $\mathbf{1 0}$.

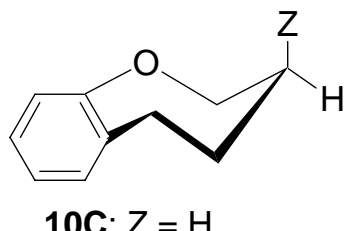

10Ca: $Z=F$, OMe

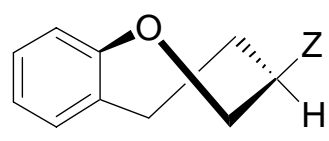

10TB: $Z=F$, OMe

The unsubstituted compound adopts a chair conformation in solution, the TB conformation not being detected. But should position 3 be substituted by an electronegative group such as $\mathrm{F}$ or $\mathrm{MeO}$, together with the chair conformation with the substituent axial, the conformation 10TB (approximately 20\%) is detected in which the methoxy group adopts an $\mathbf{i}_{\mathbf{g}}$ orientation. 
Nevertheless, the gauche effect is not enough to change the conformation TB into the most preferred one.

Finally, the isoclinal hydrogen atoms of C-2 are not equivalent. In one case, the anomeric isoclinal orientation $\left(\mathbf{i}_{\mathbf{a}}\right)$ appears in which the angle that has a value of about $60^{\circ}$ is of the C-O-C$\mathrm{H}$ type. Substitution of this hydrogen by a methyl group gives rise to steric interactions that are higher than those of an isoclinal one due to the smaller $\mathrm{C}-\mathrm{O}$ bond length and the $\mathrm{C}-\mathrm{O}-\mathrm{C}$ angle value. Instead of that, if a methoxy group is introduced, the $\mathbf{i}_{\mathbf{a}}$ is the orientation preferred because the steric interactions are smaller than those corresponding to the axial orientations and this orientation is stabilized by the anomeric effect. For the geminal hydrogen (false isoclinal, $\mathbf{i}_{\mathbf{f}}$ ) the opposite holds true: the steric interactions are lesser but a stabilization due to the anomeric effect does not take place. Again an experimental confirmation of the preference for this type of orientation is offered by 2-benzoxepine, ${ }^{2}$ whose conformational preference is the chair form. When C-3 is substituted by a methoxy group the conformation preferred is 11Ca, but the second one in importance is the 11TB one with the methoxy group in an $\mathbf{i}_{\mathbf{a}}$ orientation.

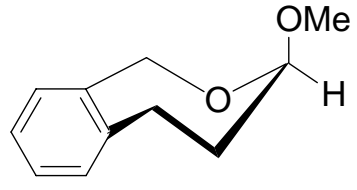

$11 \mathrm{Ca}$

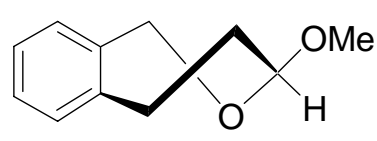

11TB

\section{Conformational Behavior of 1,4-Dioxepane Derivatives}

Once the criteria for defining the hydrogen character have been established, it is possible to turn back to our problem in 1,4-dioxepane derivatives in order to answer our previous question about the influence of the substituent on the conformational behavior of the ring.

It is possible to classify H-2, H5 and H-6 hydrogen atoms in all the 14 TC conformations of 1,4-dioxepane. ${ }^{30}$ It can be observed that a $\beta$-orientated H-5 hydrogen atom only adopts an $\mathbf{i}_{\mathbf{a}}$ disposition in the conformation TC3, whilst a $\beta$-orientated H-2 atom only acquires this orientation in TC7. In addition, the $\mathbf{i}_{\mathbf{g}}$ orientation does not exist in this molecule. Thus, the great stability and population of the TC3 conformation can be explained as a consequence of the very favored orientation that the methoxy group adopts in this conformation in the 5-methoxy-1,4dioxepane 4. On the other hand, in 2-methoxy-1,4-dioxepane 5 the $\mathbf{i}_{\mathbf{a}}$ appears only in TC7 and consequently, the change in the conformational preference of the ring takes place and TC7 becomes important in the pseudorotational equilibrium of this compound. TC3 is not the most stable conformation because of its high energy in the unsubstituted ring Finally in 6-methoxy1,4-dioxepane $\mathbf{6}$ the methoxy group adopts an axial orientation because the $\mathbf{i}_{\mathbf{g}}$ orientation does not exist in the molecule. 
<smiles>CO[C@H]1CCO[C@@H](C)CO1</smiles>

12

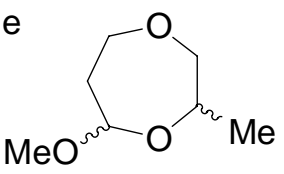

13

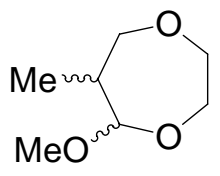

14

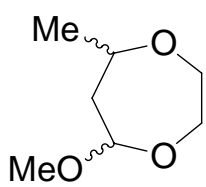

15

We extended our research on methyl-5-methoxy-1,4-dioxepanes 12-15 in order to check these ideas, studying both cis-trans isomers.

The behavior of these compounds fits two different patterns:

(a) On one hand, in one of the diastereoisomers of each compound, the TC3 conformation acquires an enormous importance (conformational population $>90 \%$ ) and is almost the only existing conformation in the compound (Figure 10). ${ }^{30}$ In it the methoxy group is $\mathbf{i}_{\mathbf{a}}$ and the methyl group is equatorial. The concordance between the theoretical coupling constants and the experimental ones is very good.

(b) On the other hand, in the other diastereoisomer, a mixture of several conformations of similar energy appears, and between them the conformation TC3 is not the most important one because in it the methyl group would adopt an axial disposition.



Figure 10. TC3 conformations of compounds $c-12, t-13, t-14$ and $c-15$. MM2 and MM3 relative energies $(\mathrm{kcal} / \mathrm{mol})$ and conformational populations (in parentheses, \%) are shown.

\section{Conclusions}

- Criteria for the nomenclature of TC conformations in seven-membered rings have been developed as a function of the values and signs of the intracyclic torsional angles.

- The influence of the presence of an $\mathrm{O}$ and $\mathrm{S}$ atom on the conformational stability of the different TC conformations as a function of the position of the two-fold pseudoaxis has been qualitatively assessed. The preferred order of stability for the $\mathrm{S}$ atom is: $\beta>\alpha>\gamma>\mathrm{S}$; while for the $\mathrm{O}$ atom is: $\alpha>\gamma>\beta>\mathrm{O}$.

- Heteroatom effects can be used to predict the stability order of the different TC conformations in heterocycles with two heteroatoms. 
- The different hydrogen atoms of oxepane have been classified according to the $\alpha$ and $\beta$ angle types. The different nature of the $\beta$ angles determines the interaction values which appear when the hydrogen atom is substituted, affecting both the steric interactions and the conformational effects that may be present.

- The steric interactions that appear when one of these hydrogen atoms is substituted vary according to the following sequence: $\mathbf{a}>\mathbf{i}_{\mathbf{a}}>\mathbf{i}=\mathbf{i}_{\mathbf{f}}>\mathbf{i}_{\mathbf{g}}$.

- The substituents whose conformational preference is governed by the anomeric effect prefer to adopt an $\mathbf{i}_{\mathbf{a}}$ orientation, in which the steric interactions are lesser than in an axial one.

- The gauche effect causes the substituents in which it appears to adopt an $\mathbf{i}_{\mathbf{g}}$ orientation.

- The anomeric effect may modify the conformational preference of the base ring. Two requirements are needed for this to happen:

- that the energy difference between the most stable conformation of the ring and that undergoing stabilization by the $\mathbf{i}_{\mathbf{a}}$ orientation is not too high so that it can be compensated for by the anomeric effect, and

- that an $\mathbf{i}_{\mathbf{a}}$ orientation between the most stable conformations of the ring does not exist.

From these studies, we hope to extend the rules presented in this account to other sevenmembered rings.

\section{References}

1. Dionne, P.; St-Jacques, M. Can. J. Chem. 1989, 67, 11.

2. Désilets, S.; S-Jacques, M. J. Am. Chem. Soc. 1987, 109, 1641.

3. Canuel, L.; St-Jacques, M. Can. J. Chem. 1974, 52, 3581.

4. (a) Gianni, M. H.; Adams, M.; Kuivila, H. G.; Wurthorn, K. J. Org. Chem. 1975, 40, 450.

(b) St-Amour, R.; St-Jacques, M. Can. J. Chem. 1981, 59, 2283.

5. St-Amour, R.; Phan Viet, M. T.; St-Jacques, M. Can. J. Chem. 1984, 62, 2830.

6. Lachapelle, A.; St-Jacques, M. Tetrahedron 1988, 44, 5044.

7. (a) Ménard, D.; St-Jacques, M. Can. J. Chem. 1981, 59, 1160. (b) Ménard, D.; St-Jacques, M. J. Am. Chem. Soc. 1984, 106, 2055. (c) Dionne, P.; St-Jacques, M. J. Am. Chem. Soc. 1987, 109, 2616.

8. (a) Blanchette, A.; Sauriol-Lord, F.; St-Jacques, M. J. Am. Chem. Soc. 1978, 100, 4055. (b) St-Amour, A.; St-Jacques, M. Can. J. Chem. 1983, 61, 109.

9. (a) Ermer, O. Aspekte von Keftfeldrechmungen: Wollfgang Bauer Verlag: Múnchen, Germany, 1981. (b) Ōsawa, E.; Musso, H. Top. Stereochem. 1982, 13, 117. (c) Burkert, U.; Allinger, N. L. Molecular Mechanics; ACS Monograph No. 177, 1982. (d) Ōsawa, E.; Musso, H. Angew. Chem., Int. Ed. 1983, 22, 1. 
10. (a) Hendrickson, J. B. J. Am. Chem. Soc. 1967, 89, 7036. (b) Bocian, D. F.; Picket, M. M.; Rounds, T. C.; Strauss, H. L. J. Am. Chem. Soc. 1975, 97, 687. (c) Ōsawa, E.; Musso, H. J. Comput. Chem. 1984, 5, 307.

11. Ermolaeva, L. I.; Mastryukov, V. S.; Allinger, N. L.; Almenningen, A. J. Mol. Struct. 1989, $196,151$.

12. Espinosa, A.; Gallo, M. A.; Entrena, A.; Gómez, J. A. J. Mol. Struct. 1994, 323, 247.

13. Espinosa, A.; Gallo, M. A.; Entrena, A.; Campos, J.; Domínguez, J. F.; Camacho, E.; Sánchez, I. J. Mol. Struct. 1993, 296, 133.

14. Espinosa, A.; Gallo, M. A.; Entrena, A.; Gómez, J. A. J. Mol. Struct. 1994, 323, 247.

15. (a) Bocian, D. F.; Strauss, H. L. J. Am. Chem. Soc. 1977, 99, 2876. (b) Kamalov, G. L.; Kuzmin, V. E.; Sharigin, V. N.; Verkhivker, G. M. Vopr. Stereokhim. 1984, 7, 72.

16. Entrena, A.; Jaime, C.; Espinosa, A.; Gallo, M. A. J. Org. Chem. 1989, 54, 1745.

17. Entrena, A.; Gallo, M. A.; Espinosa, A.; Jaime, C.; Campos, J.; Domínguez, J. F. J. Org. Chem. 1989, 54, 6034.

18. (a) Juaristi, E.; Cuevas, G. The Anomeric Effect; CRC Press: Boca Raton, Florida, 1995. (b) Eliel, E. L.; Wilen, S. H. Stereochemistry of Organic Compounds; John Wiley \& Sons, Inc: New York, 1994.

19. (a) Abe, A. J. Am. Chem. Soc. 1976, 98, 6477. (b) Both, H.; Grindely, T. B.; Kuedhair, K. A. J. Chem. Soc. Chem. Commun. 1982, 1047.

20. (a) Wolfe, S. Acc. Chem. Res. 1972, 5, 102. (b) Juaristi, E. J. Chem. Educ. 1979, 56, 438. (c) Zefirov, N. S.; Gurvich, L. G.; Shashkov, A. E.; Krimer, H. Z.; Vorobeva, E. A. Tetrahedron 1976, 32, 1211.

21. (a) Connor, T. M.; Mac Laugchlan, K. A. J. Phys. Chem. 1965, 69, 1888. (b) Snyder, R. G.; Zerbi, G. Spectrochim. Acta 1967, 23A, 391.

22. Mark, J. E.; Flory, P. J. J. Am. Chem. Soc. 1965, 87, 1966; 1966, 88, 3792.

23. Abraham, R. J.; Banks, H. D.; Eliel, E. L.; Hofer, O.; Kaloustian, M. K. J. Am. Chem. Soc. 1972, 94, 1913

24. Allinger, N. L. J. Am. Chem. Soc. 1977, 99, 8127,

25. (a) Allinger, N. L.; Yuh, Y. H.; Lii, J. H. J. Am. Chem. Soc. 1989, 111, 8551. (b) Lii, J. H.; Allinger, N. L. J. Am. Chem. Soc. 1989, 111, 8566. (c) Lii, J. H.; Allinger, N. L. J. Am. Chem. Soc. 1989, 111, 8576.

26. Dillen, J.; Geise, H. J. J. Chem. Phys. 1979, 70, 425.

27. Hendrickson, J. B. J. Am. Chem. Soc. 1964, 86, 4854.

28. (a) Díez, E.; Esteban, A. L.; Guilleme, J.; Bermejo, F. J. J. Mol. Struct. 1981, 70, 61. (b) Esteban, A. L.; Galiano, C.; Díez, E.; Bermejo, F. J. J. Chem. Soc., Perkin Trans. 2 1982, 657.

29. Cano, F. H.; Foces-Foces, C. J. Mol. Struct. 1983, 94, 209.

30. Entrena, A.; Campos, J.; Gómez, J. A.; Gallo, M. A.; Espinosa, A. J. Org. Chem. 1997, 62, 337. 
31. Espinosa, A.; Entrena, A.; Gallo, M.A.; Campos, J. Domínguez, J.F.; Camacho, E.; Garrido, R. J. Org. Chem. 1990, 55, 6018.

32. Hendrickson, J. B. J. Am. Chem. Soc. 1967, 89, 7043.

33. Bucourt, R. Top. Stereochem. 1974, 8, 159.

34. (a) Booth, H.; Everett, J. J. Chem. Soc., Chem. Commun. 1976, 278. (b) Schneider, H. J.; Hoppen, V. Tetrahedron Lett. 1974, 579. 